\title{
Optical isolation in topological-edge-state photonic arrays
}

\author{
Ramy El-Ganainy* and Miguel Levy \\ Department of Physics, Michigan Technological University, Houghton, Michigan 49931, USA \\ *Corresponding author: ganainy@mtu.edu
}

Received 15 September 2015; revised 11 October 2015; accepted 12 October 2015; posted 14 October 2015 (Doc. ID 250060); published 6 November 2015

We introduce a new type of optical isolator based on breaking time reversal symmetry in dissipative finite Su-Schrieffer-Heeger (SSH) waveguide arrays that support topological edge states at one end of the structure. In the forward propagation direction, light is launched into the edge waveguide to excite the localized topological midgap state. As a result, most of the input optical power is transmitted to the output port. On the other hand, backward reflected light encounters a propagation constant mismatch in that same channel which shifts the otherwise midgap state into one of the bands and hence becomes delocalized over the whole array. We show that under these conditions, a judicious spatial distribution of the optical dissipation across the structure can produce an isolation ratio of $-50 \mathrm{~dB}$. The required nonreciprocal phase shift is introduced by depositing a magnetic garnet film only on the edge waveguide and, thus, the required magnetic field can be generated by an integrated micromagnet. Similar concepts can also be applied to SSH arrays made from optical resonators. () 2015 Optical Society of America

OCIS codes: (230.3240) Isolators; (230.7370) Waveguides; (230.3810) Magneto-optic systems.

http://dx.doi.org/10.1364/OL.40.005275

Topology is a branch of mathematics that studies certain general properties of objects, rather than their geometrical details. In particular, it deals with those features that do not change under a certain class of continuous deformation [1]. The discovery that certain condensed matter materials can support topologically protected defect states has launched an intensive research program that focuses on investigating these structures and their potential applications [2]. In two important papers by Haldane and Raghu, this work was extended to optics where a photonic realization of quantum edge Hall states was theoretically proposed $[3,4]$. Shortly after, these predictions were theoretically extended and experimentally verified by using photonic crystals operating in the microwave regime [5,6]. This work has stirred intensive activities in the field of topological photonics [7-12]. An important feature of topological states is their robustness against disorder. This allows for building photonic devices that are more immune to fabrication errors. We note, however, that due to the relative weak magnetic effects at optical frequencies, work on magneto-optical topological structures has been very limited. Given that a true breaking of time reversal symmetry in optics is usually achieved by using magneto-optical effects, it is important to explore this domain.

Here we consider a certain class of defect states in onedimensional waveguide arrays with interleaving coupling coefficients, as shown schematically in the top panel of Fig. 1. This structure, also known as $\mathrm{Su}-\mathrm{Schrieffer-Heeger} \mathrm{(SSH),} \mathrm{has} \mathrm{been}$ investigated before in the context of polymer science [13]. It has also been shown that localized edge states can be engineered by terminating the array with weak coupling, as shown in the middle panel of Fig. 1. On the other hand, defect bulk states can be created by the arrangement shown in the bottom panel of Fig. 1. More interestingly, these states have been proven to be of topological nature and to be immune against off-diagonal

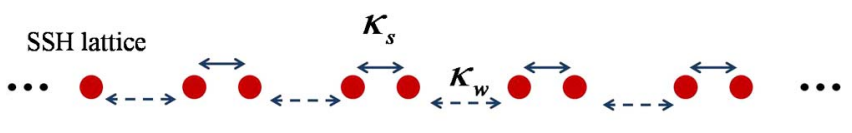

Topological edge state

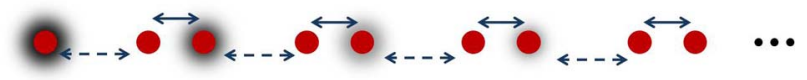

Topological bulk state

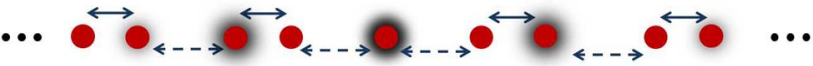

Fig. 1. Schematic of the coupling profile in an infinite SSH lattice (top panel), topological edge state in semi-infinite SSH array (middle panel), and topological bulk state in a hetero-structure SSH configuration. The red circles represent waveguides or cavities, while the strong and weak coupling links are represented by solid (short) and broken (long) arrows, respectively. The mode profiles in the middle and lower panels are indicated schematically by the halos around the circles with a darker halo equivalent to stronger amplitude. Note that the modal amplitude is zero at each second site (counting from the site of maximum field). 
perturbation, namely perturbation introduced to the coupling coefficients between the waveguides [14]. We note that Hermitian [15], non-Hermitian [16-18], and PT symmetric [19] SSH optical structures have been recently investigated by different research groups.

In this Letter, we focus on a waveguide array similar to the schematic arrangement shown in the middle part of Fig. 1. Furthermore, we assume that a magnetic garnet film is deposited on top of the leftmost waveguide element [20]. By applying an external static magnetic field in the transverse direction and by considering only TM waveguide modes, it is straightforward to show that the propagation constant of that edge waveguide will have different forward and backward values [20]. Within the formalism of coupled mode theory (CMT), the system can be described by

$$
\begin{aligned}
i \frac{d \vec{a}_{f, b}}{d z} & =H_{f, b} \vec{a}_{f, b}, \\
H_{f, b} & =\left(\begin{array}{ccccc}
\beta_{f, b} & \kappa_{w} & 0 & 0 & 0 \\
\kappa_{w} & \beta-i \gamma_{2} & \kappa_{s} & \vdots & 0 \\
\vdots & \vdots & \vdots & \vdots & 0 \\
0 & 0 & \kappa_{w} & \beta-i \gamma_{N-1} & \kappa_{s} \\
0 & 0 & 0 & \kappa_{s} & \beta-i \gamma_{N}
\end{array}\right) .
\end{aligned}
$$

In Eq. (1), $z$ is the propagation distance, and the elements of the vector $\vec{a}=\left[\begin{array}{llll}a_{1} & a_{2} & \cdots & a_{N}\end{array}\right]^{T}$ represent the optical field amplitude in the individual waveguides with the superscript $T$ indicating matrix transpose and $N$ being the total number of waveguides. The first waveguide exhibits different propagation constants $\beta_{f, b}$ in the forward and backward propagation directions, respectively, while all other waveguides have a fixed propagation constant $\beta$ in both directions. In addition, we assume that all waveguides, except the first one, also exhibit optical loss that can be characterized by the coefficients $\gamma_{n}$ where $n=1,2,3, \ldots N$ are the waveguide numbers from left to right. The weak and strong values of the coupling coefficients are represented by $\kappa_{w, s}$. Note that, if the semi-infinite array satisfies the conditions $\beta_{f}=\beta$ and $\gamma_{n}=0$ for all $n$, then the field amplitude associated with the topological edge state can be expressed analytically as $a_{2 m-1}=(-r)^{m-1} \sqrt{1-r^{2}}$ and $a_{2 m}=0$, where $r=\kappa_{w} / \kappa_{s}$ and $m=1,2,3, \ldots$ Interestingly these field amplitudes vanish at each second waveguide. As we will see, this feature persists, even when the array is finite, and will be very crucial for the proposed optical isolator structure.

For illustration purposes, we consider the specific case of seven waveguides, and we assume $\kappa_{w} / \kappa_{s}=0.2$. We also assume that $\beta_{f}=\beta, \gamma_{n} / \kappa_{s}=0.3$ for $n=2,4,6$ and zero otherwise. The losses can be introduced by depositing periodic metallic stripes on top of the waveguides, and the average loss can be tuned by engineering the duty cycle of these periodic stripes. Absorption losses can also be introduced into silicon waveguides by implantation doping [21]. A schematic of optical implementation of the photonic structure under investigation is shown in the top panel of Fig. 2. It consists of evanescently coupled waveguide channels with an interleaving channel-to-channel separation to produce the strong/weak coupling profile. A magnetic garnet film is deposited only on the

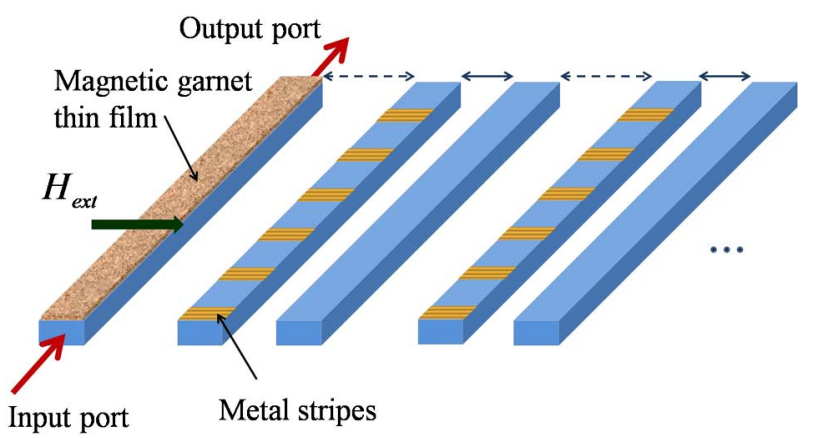

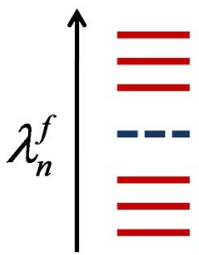

Forward propagation

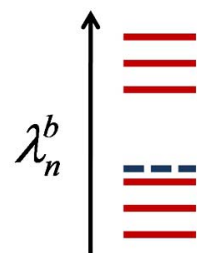

Backward propagation
Fig. 2. Schematic of the photonic structure under investigation is shown in the top panel. It consists of evanescently coupled waveguide channels with interleaving coupling strength channel-to-channel separation to produce the strong/weak coupling profile. A magnetic garnet film is deposited only on the first waveguide element. Under the application of an external static magnetic field, the optical mode of this waveguide will experience a different propagation constant in the forward and backward propagation directions. The losses can be introduced by depositing periodic metallic stripes, and the average loss can be tuned by engineering the duty cycle of these periodic stripes. The eigenvalue spectra of forward and backward propagating modes in the absence of any optical absorption are depicted in the lower panel. In the forward direction, the topological state (shown in a blue dotted line) is localized, and its field amplitude vanishes every second channel. In the backward propagation direction, time reversal symmetry is broken due to the gyrotropy of the magnetic garnet film, and the eigenvalue of the otherwise midgap state is now pushed toward the lower band. As a result, the corresponding mode is delocalized and suffers losses.

first waveguide element. Under the application of a transverse external static magnetic field, the TM optical mode of this waveguide will experience different propagation constants in the forward and backward propagation directions [22]. The eigenvalue spectra of forward and backward propagating modes in the absence of any optical absorption are depicted in the lower panel.

Based on our discussion and, since the field amplitudes of the topological edge mode have small values (almost zero) in the even waveguide channels, these optical losses are not expected to have a significant impact on the forward propagating light. Figure 3(a) confirms this prediction, where $92 \%$ of the optical intensity launched in the leftmost waveguide can propagate intact to the output with most of the missing $8 \%$ coupled to the odd channels with minimum impact from the even waveguide elements.

We now consider the backward propagation dynamics, and we assume that the gyrotropic effects are engineered [22] such that $\beta_{b}=\beta-\kappa_{s}$. The eigenvalue spectrum is shown schematically in Fig. 2 . Note that now the otherwise midgap state is pushed into the discrete band. As a result, the corresponding 


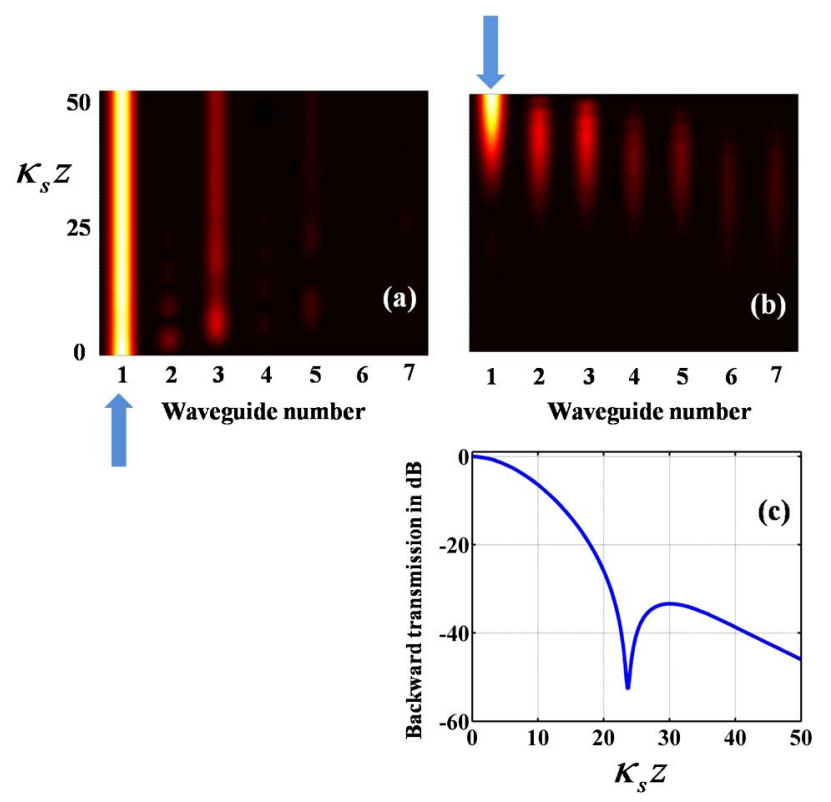

Fig. 3. Numerical simulations of the field amplitudes associated with light propagation in a truncated magneto-optical SSH photonic array similar to that described in Fig. 2 when (a) light is launched in the leftmost waveguide in the forward direction and (b) same as in (a) but for backward propagation where $\beta_{b}=\beta-\kappa_{s}$. The total number of waveguides in the array is seven. The backward transmission as a function of the array length is depicted in (c). Evidently, isolation ratios better than $-50 \mathrm{~dB}$ can be achieved after a propagation distance of $24 / \kappa_{s}$.

state is no longer localized. Thus, any backward propagating light into the leftmost waveguide interacts with the collective delocalized optical modes of the array and suffers significant optical losses. This is demonstrated in Fig. 3(b). Figure 3(c) depicts the dynamics of the backward propagation only in the left-most waveguide in logarithmic scale. Evidently, isolation ratios better than $-50 \mathrm{~dB}$ between forward and backward propagating light can be achieved after a propagation distance of $24 / \kappa_{s}$. Interestingly, we also observe from Fig. 3(c) that the isolation ratio (as a function of propagation distance) has a sharp dip, after which it decreases again briefly before it starts to rise again. This interesting behavior can be attributed to interplay between interference effects and optical absorption. More specifically, the intensity profile resulting from discrete diffraction will maximize the concentration of the optical intensity at the lossy waveguides at this specific length where the dip is observed, leading to higher isolation ratios. In addition, interference effects due to the finite array size can also be observed for long enough propagation distances.

To confirm our results obtained by using coupled mode analysis, we employ beam propagation method (BPM) simulation to examine a realistic structure made up of a sevenwaveguide array consisting of $300 \mathrm{~nm}$ thick $1 \mu \mathrm{m}$ wide silicon waveguide channels on insulator (SOI). Figure 4 depicts one of these channels.

The leftmost waveguide contains a cerium-substituted yttrium iron garnet (Ce:YIG) cover layer, which yields a nonreciprocal phase shift $\Delta \beta^{(n r)}=\beta_{b}-\beta_{f}=-35 \mathrm{~cm}^{-1}$ at a telecom wavelength of $1.55 \mu \mathrm{m}$ [22]. This, however, will introduce

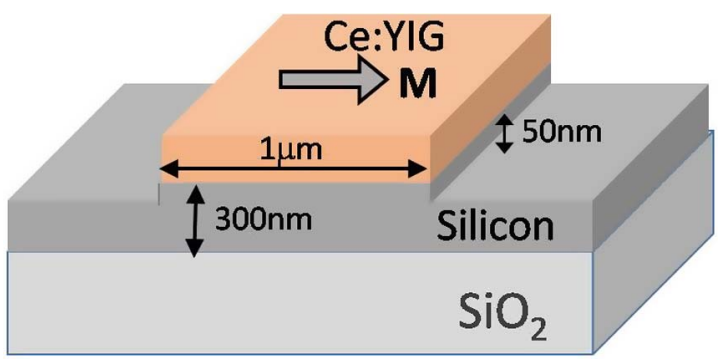

Fig. 4. Waveguide structure of an individual channel of the array. The leftmost waveguide has Ce:YIG as a cover layer. Its magnetization vector is denoted by $\mathbf{M}$. All other waveguide channels have the same structure, except for an index matched nonmagnetic cover layer having a similar refractive index to Ce:YIG.

a phase mismatch between that first waveguide and the rest of the channels, even in a forward propagation direction. To maintain the phase-matching condition $\beta_{f}=\beta$, one can either optimize the dimensions of the other guiding channels or deposit on each of them a nonmagnetic cover layer that is index matched to Ce:YIG to equalize the forward propagation constants. Note that there are a number of compounds with refractive indices close to Ce:YIG, such as titanium dioxide (rutile) and tellurium dioxide. Moreover, the garnet index can also be tuned by adjusting the Ce substitution level. In our simulations, we assume that each of the nonmagnetic waveguides is covered with one of these phase-matching layers.

To adjust the strong coupling between the waveguides to be $\kappa_{s}=-\Delta \beta^{(n r)}=35 \mathrm{~cm}^{-1}$, their center-to-center separation is chosen to be $1.97 \mu \mathrm{m}$. On the other hand, the weak coupling $\kappa_{w} / \kappa_{s}=0.2$, can be satisfied with a center-to-center separation of $2.42 \mu \mathrm{m}$.

Figures 5(a) and 5(b) depict the propagation dynamics in both the forward and backward directions for such an array, respectively. The simulations are performed by using semi-vectorial beam propagation simulation for an input TM mode at $1.55 \mu \mathrm{m}$. The absorption losses corresponding to $\gamma_{2,4,6} / \kappa_{s}=$ 0.3 were introduced into waveguides 2,4 , and 6 , by adding a $3.5 \times 10^{-4}$ imaginary component to the silicon channel refractive index. Clearly, the results obtained by analyzing this realistic structure using the BPM are in excellent agreement with the conclusions of our coupled mode analysis. In particular, we find that an isolation ratio (defined as the power ratio between the return power into waveguide 1 and the forward transmission of the device) of $-50 \mathrm{~dB}$ can be achieved for a device length of $z=7.35 \mathrm{~mm}$.

Note that, in general, the device length can be reduced by further increasing the coupling strength between the waveguide elements. However, this would require a stronger gyrotropy. Thus, it is clear that the same configuration can be scaled depending on the application. We note that our simulations indicate that the isolation ratio drops to $-15 \mathrm{~dB}$ if the backward propagation constant deviates by $0.1 \kappa_{s}$ from its ideal value for the same device length. This drawback can be overcome either by fabricating a longer device to allow for the signal to attenuate further or by adjusting the applied magnetic field to tune the backward propagation constant to its optimal value.

Finally, we compare the isolator structure proposed in this Letter with that introduced in $[23,24]$. While the coherent 


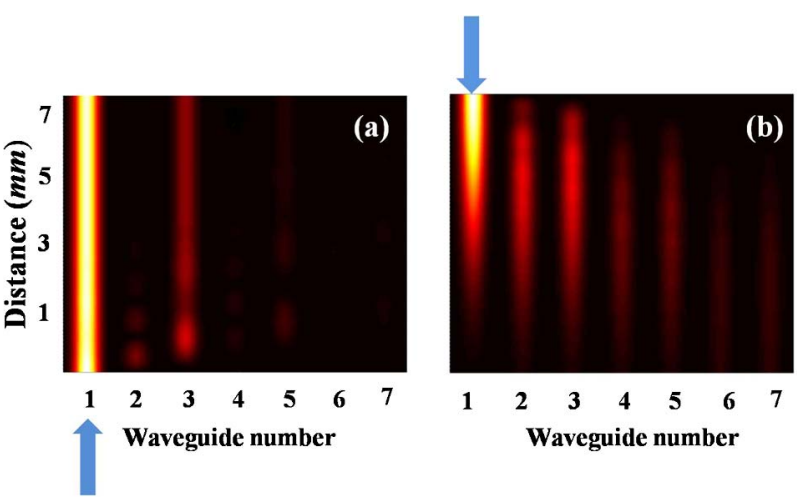

Fig. 5. Field amplitudes of forward (a) and backward (b) propagation dynamics in a waveguide array similar to that depicted in Fig. 2 when the individual channels are made of waveguides similar to that described in Fig. 4. The center-to-center separation between the waveguides is alternating between 1.97 and $2.42 \mu \mathrm{m}$ to adjust the strong and weak coefficients to $\kappa_{s}=35 \mathrm{~cm}^{-1}$ and $\kappa_{w} / \kappa_{s}=0.2$. The simulations are performed by using a semi-vectorial BPM and indicate that an isolation ratio of $-50 \mathrm{~dB}$ is possible after a propagation distance of $7.35 \mathrm{~mm}$. This in good agreement with the results obtained from the coupled mode theory which predicts the same behavior after a propagation distance of $\sim 6.8 \mathrm{~mm}$. This small discrepancy is due to the fact that the BPM accounts for more realistic effects that are not considered in $\mathrm{CMT}$.

transport and surface Bloch oscillations in the magneto-optical array presented in $[23,24]$ allows for higher isolation ratios, the design and fabrication of that array requires a careful tuning of the coupling coefficients that vary from one adjacent waveguide pair to another. In addition, the gyrotropy must be introduced on all waveguides. In contrast, the isolator proposed in the current work provides a smaller isolation, but offers a more straightforward design and implementation. Even more importantly, the gyrotropy is introduced only on the first guiding channels which can be achieved by integrating a small micro-magnet on the same chip [25].

In conclusion, we have proposed a new type of optical isolator based on breaking time reversal symmetry of topological defect states in dissipative SSH photonic arrays. In particular, we have shown that when light propagating in the forward direction is launched into the leftmost waveguide, the intensity transmission is about $92 \%$. On the other hand, a judicious design of the nonreciprocal phase shift and the optical absorption of the array can result in a high isolation ratio for backward propagating light. These nonreciprocal effects can be introduced by using magnetic garnet films on top of the leftmost waveguide while the optical losses can be controlled by depositing lossy metallic layers on top of the guiding channels. Our numerical results based on the coupled mode theory and BPM are in good agreement and predict that for realistic values of the nonreciprocal phase shift of $-35 \mathrm{~cm}^{-1}$, an isolation ratio of $-50 \mathrm{~dB}$ can be achieved when the device length is $\sim 7 \mathrm{~mm}$. Moreover, since the magneto-optical effects in our proposed structure are introduced only on the leftmost waveguide, they can be generated by using integrated micromagnets. It is noteworthy that similar concepts to those proposed here can also be applied to SSH photonic arrays made of optical resonators. Finally, we note that the isolator proposed here functions only for TM waves, whereas TE waves will experience identical forward and backward propagation dynamics.

\section{REFERENCES}

1. M. Nakahara, Geometry, Topology and Physics (CRC Press, 2003).

2. M. Z. Hasan and C. L. Kane, Rev. Mod. Phys. 82, 3045 (2010).

3. F. D. M. Haldane and S. Raghu, Phys Rev. Lett. 100, 013904 (2008)

4. S. Raghu and F. D. M. Haldane, Phys. Rev. A 78, 033834 (2008).

5. Z. Wang, Y. D. Chong, J. D. Joannopoulos, and M. Soljacic, Phys. Rev. Lett. 100, 013905 (2008).

6. Z. Wang, Y. Chong, J. D. Joannopoulos, and M. Soljacic, Nature 461, 772 (2009).

7. M. Hafezi, E. A. Demler, M. D. Lukin, and J. M. Taylor, Nat. Phys. 7, 907 (2011).

8. K. Fang, Z. Yu, and S. Fan, Nat. Photonics 6, 782 (2012).

9. Y. E. Kraus, Y. Lahini, Z. Ringel, M. Verbin, and O. Zilberberg, Phys. Rev. Lett. 109, 106402 (2012).

10. M. C. Rechtsman, J. M. Zeuner, Y. Plotnik, Y. Lumer, D. Podolsky, F. Dreisow, S. Nolte, M. Segev, and A. Szameit, Nature 496, 196 (2013).

11. M. Hafezi, S. Mittal, J. Fan, A. Migdall, and J. M. Taylor, Nat. Photonics 7, 1001 (2013).

12. L. Lu, J. D. Joannopoulos, and M. Soljacic, Nature 8, 821 (2014).

13. W. P. Su, J. R. Schrieffer, and A. J. Heeger, Phys. Rev. B 22, 2099 (1980).

14. S. Ryu and Y. Hatsugai, Phys. Rev. Lett. 89, 077002 (2002).

15. N. Malkova, I. Hromada, X. Wang, G. Bryant, and Z. Chen, Opt. Lett. 34, 1633 (2009).

16. T. Eichelkraut, R. Heilmann, S. Weimann, S. Stützer, F. Dreisow, D. N. Christodoulides, S. Nolte, and A. Szameit, Nat. Commun. 4, 2533 (2013).

17. J. M. Zeuner, M. C. Rechtsman, Y. Plotnik, Y. Lumer, S. Nolte, M. S. Rudner, M. Segev, and A. Szameit, Phys. Rev. Lett. 115, 040402 (2015).

18. C. Poli, M. Bellec, U. Kuhl, F. Mortessagne, and H. Schomerus, Nat. Commun. 6, 6710 (2015).

19. H. Schomerus, Opt. Lett. 38, 1912 (2013).

20. M. Levy and P. Kumar, Opt. Lett. 35, 3147 (2010).

21. P. E. Schmid, Phys. Rev. B 23, 5531 (1981).

22. H. Yokoi, Y. Shoji, and T. Mizumoto, Jpn. J. Appl. Phys. 43, 5871 (2004).

23. R. El-Ganainy, A. Eisfeld, M. Levy, and D. N. Christodoulides, Appl. Phys. Lett. 103, 161105 (2013).

24. R. El-Ganainy and M. Levy, IEEE Photon. J. 6, 1 (2014).

25. M. Levy, H. Hegde, F. J. Cadieu, R. Wolfe, V. J. Fratello, and R. M. Osgood, Jr., IEEE Photon. Technol. Lett. 8, 903 (1996). 